\title{
Bounded Traveling Wave Solutions of the (3+1)-Dimensional Zakharov- Kuznetsov Equation with Power Law Nonlinearity \\ Li Wei*, Minrong Ren
}

College of Applied Mathematics, Chengdu University of Information Technology, Chengdu 610025, Sichuan, P. R. China

DOI: $10.36347 /$ sipms.2020.v07i07.004

| Received: 15.07.2020 | Accepted: 23.07.2020 | Published: 26.07.2020

*Corresponding author: Li Wei

Abstract

Review Article

In this paper, the bifurcation theory of dynamical system is applied to study the traveling waves of the $(3+1)$ dimensional Zakharov-Kuznetsov Equation with Power Law Nonlinearity. By transforming the traveling wave system of the Zakharov-Kuznetsov equation into a dynamical system in $R^{2}$, we derive various parameter conditions which guarantee the existence of its bounded and unbounded orbits. Furthermore, by calculating complicated elliptic integrals along these orbits, we obtain exact expressions of bounded traveling wave solutions of the (3+1)-dimensional Zakharov-Kuznetsov equation for $n=1$.

Keywords: (3+1)-dimensional Zakharov-Kuznetsov, bifurcation of traveling waves, dynamical system, elliptic integral.

Copyright @ 2020: This is an open-access article distributed under the terms of the Creative Commons Attribution license which permits unrestricted use, distribution, and reproduction in any medium for non-commercial use (NonCommercial, or CC-BY-NC) provided the original author and source are credited.

\section{INTRODUCTION}

The ZK equation is a very attractive model equation for the study of vortices in geophysical flows. The ZK equation appears in many areas of physics, applied mathematics and engineering. In particular, as an important model to describe nonlinear ion acoustic waves in a strongly magnetized lossless plasma composed of cold ions and hot isothermal electrons, traveling waves of the $(3+1)$-dimensional ZakharovKuznetsov equation have been concerned widely.

In 2010, by means of the classical and the associated vector fields method, Dong et al. derived the reductions and some new solutions of the $(3+1)$ dimensional Zakharov-Kuznetsov equation [1]. In 2012, Zhang and Zhou [2] obtained the bounded solitary wave, periodic, kink and antikink solutions to the $\mathrm{ZK}$ equation in general form by employing the bifurcation theory. In 2013, Wronskian form expansion method was applied in [3] to obtain new interaction solutions of (3+1)-dimensional Zakharov-Kuznetsov equation. Later, by using improved fractional sub equation method, S. Sahoo and S. Saha Ray constructed the analytical exact solutions of (3+1)-dimensional generalized fractional KdV-Zakharov-Kuznetsov equations [4]. In 2016, Moleleki et al., proposed Kudryashov and Jacobi elliptic function method to construct exact solutions of the $(3+1)$-dimensional Zakharov-
Kuznetsov equation [5]. In 2017, Lu et al., used modified extended direct algebraic method to obtain new exact solitary wave, soliton and elliptic function solutions of $(3+1)$-dimensional nonlinear extended Zakharov-Kuznetsov and modified $\mathrm{KdV}$-ZakharovKuznetsov equations [6]. In the same year, by using the modified form of the Kudryashov method, exact solutions to conformable time fractional $(3+1)$ dimensional equations were derived [7]. In 2018, Wang et al. used modified extended mapping method to get exact traveling wave solutions of different forms such as bright and dark solitary wave, periodic solitary wave, dark and bright soliton, etc [8].

In this paper, we consider the $(3+1)$ dimensional Zakharov-Kuznetsov equation with Power Law Nonlinearity

$$
U_{t}+a U^{n} U_{x}+b\left(U_{x x}+U_{y y}+U_{z z}\right)_{x}=0,(1.1)
$$

Where $a, b$ are constants our aim is to completely investigate all traveling waves of Eq (1.1) by the bifurcation method of dynamical system [9-12] and give explicit expressions of bounded wave solutions.

Traveling wave system and bifurcation analysis With the following traveling wave transformation

$$
U=U(x, y, z, t)=u(\xi)=u(x+y+z-c t),
$$


Equation (1) can be transformed into its raveling wave system

$$
-c u^{\prime}+a u^{n} u^{\prime}+3 b u^{\prime \prime \prime}=0
$$

Where' stands for $d / d \xi$ and $c \neq 0$ is the wave speed. Integrating (2.1) once and retaining an integral constant, we have

$$
-c u+\frac{a}{n+1} u^{n+1}+3 b u^{\prime \prime}=e
$$

Where parameter $e$ is the integral constant, equation (2.2) has the following equivalent form

$$
\left\{\begin{array}{c}
u^{\prime}=v, \\
v^{\prime}=\frac{1}{3 b}\left(-\frac{a}{n+1} u^{n+1}+c u+e\right)
\end{array}\right.
$$

Which is a dynamical system in $R^{2}$. which is exactly a Hamiltonian system with the energy function $H(u, v)=\frac{1}{2} v^{2}+\frac{1}{3 b}\left(\frac{a}{(n+1)(n+2)} u^{n+2}-\frac{c}{2} u^{2}-e u\right)$

When $\mathrm{n}=1$, system convert (2.3) the following form

$$
\left\{\begin{array}{c}
u^{\prime}=v, \\
v^{\prime}=\frac{1}{3 b}\left(-\frac{a}{2} u^{2}+c u+e\right),
\end{array}\right.
$$

Firstly, we start with equilibria of system (2.5).

Theorem2.1. When $c^{2}+2 a e>0$, system (2.5) has two equilibrium, a saddle $P_{1}\left(\frac{c-\sqrt{c^{2}+2 a e}}{a}, 0\right)$, and a center $P_{2}\left(\frac{c+\sqrt{c^{2}+2 a e}}{a}, 0\right)$. When $c^{2}+2 a e=0$, system (2.5) has a unique cusp $P_{3}\left(\frac{c}{a}, 0\right)$. When $c^{2}+2 a e<0$, system (2.5) has no equilibrium.

Proof. When $c^{2}+2 a e>0$, a direct calculation shows that system (2.5) has two equilibrium

$P_{1}\left(\frac{c-\sqrt{c^{2}+2 a e}}{a}, 0\right), \quad P_{2}\left(\frac{c+\sqrt{c^{2}+2 a e}}{a}, 0\right) . \quad$ Letting $M\left(P_{i}\right)(i=1,2,3)$ denote the Jacabian matrix of system (2.5) at an equilibrium $P_{i}$, we have

$$
\begin{gathered}
M\left(P_{1}\right)=\left[\begin{array}{cc}
\frac{0}{c^{2}+2 a e} & 1 \\
3 b & 0
\end{array}\right], \\
M\left(P_{2}\right)=\left[\begin{array}{cc}
0 & 1 \\
-\frac{\sqrt{c^{2}+2 a e}}{3 b} & 0
\end{array}\right] .
\end{gathered}
$$

It is not difficult for one to check that their determinants are

$$
\begin{gathered}
\operatorname{det} M\left(P_{1}\right)=-\frac{\sqrt{c^{2}+2 a e}}{3 b}<0, \\
\operatorname{det} M\left(P_{2}\right)=\frac{\sqrt{c^{2}+2 a e}}{3 b}>0 .
\end{gathered}
$$

By the theory of plane dynamic system [13-15] and the properties of Hamiltonian system (32), it is not difficult to check that $P_{1}$ is a saddle and $p_{2}$ is a center in this case.
When $c^{2}+2 a e=0$, the system (2.4) has an unique equilibrium $P_{3}\left(\frac{c}{a}, 0\right)$ with a nilpotent matrix

$$
M\left(P_{3}\right)=\left(\begin{array}{ll}
0 & 1 \\
0 & 0
\end{array}\right)
$$

It means that $P_{3}$ is a degenerated equilibrium. In order to be able to further determine the type of $P_{3}$, we do the following homeomorphic transformation

$$
\alpha=u-\frac{c}{a}, \beta=v,
$$

Which transforms system (2.5) into its normal form

$$
\left\{\begin{array}{l}
\alpha^{\prime}=\beta, \\
\beta^{\prime}=-\frac{a}{6 b} \alpha^{2} .
\end{array}\right.
$$

By the qualitative theory of differential equation [33 Theorem 7.3, Chapter 2], we have $k=2$ and $b_{n}=0$, which indicates that $P_{3}$ is a cusp.

When $c^{2}+2 a e<0$, it is easy to see that there is no equilibrium of system (2.5).

Next we need to illustrate the parameter bifurcation sets with $\left\{(a, c, e) \mid c^{2}+2 a e>0\right\}, \quad\left\{(a, c, e) \mid c^{2}+2 a e=0\right\}$ and $\left\{(a, c, e) \mid c^{2}+2 a e<0\right\}$.

Based on the analysis of the equilibrium and the properties of the Hamiltonian system [19], we have the following results.

\section{Case 1}

Consider $c^{2}+2 a e>0$, there is a homoclinic orbit $\gamma$ connected to the saddle $P_{1}$. The center $P_{2}$ is surrounded by the family of periodic orbits

$$
\Gamma(h)=\left\{H(p, q)=h, h \in\left(h\left(P_{2},\right), h\left(P_{1},\right)\right)\right\},
$$


Where

$$
\begin{aligned}
& h\left(P_{1}\right)=\frac{-c^{3}+\left(c^{2}+2 a e\right) \sqrt{c^{2}+2 a e}-3 a e c}{9 a^{2} b}, \\
& h\left(P_{2}\right)=\frac{-c^{3}-\left(c^{2}+2 a e\right) \sqrt{c^{2}+2 a e}-3 a e c}{9 a^{2} b} .
\end{aligned}
$$

Moreover, $\Gamma(h)$ tends to $P_{2}$ as $h \rightarrow h\left(P_{2}\right)$ and tends to $\gamma$ as $h \rightarrow h\left(P_{1}\right)$, besides the homoclinic orbit and periodic orbits, other orbits of system (2.5) are unbounded, as shown in figure-1(a).
Case 2

Consider $c^{2}+2 a e=0$, the system (2.5) has two types of orbits, of which orbit $L$ was different from other orbits, but all the orbits here were unbounded, as show in Figure-1(b).

\section{Case 3}

Consider $c^{2}+2 a e<0$, system (2.5) has only one type of orbits, and each orbit is unbounded, as show in Figure-1(c).

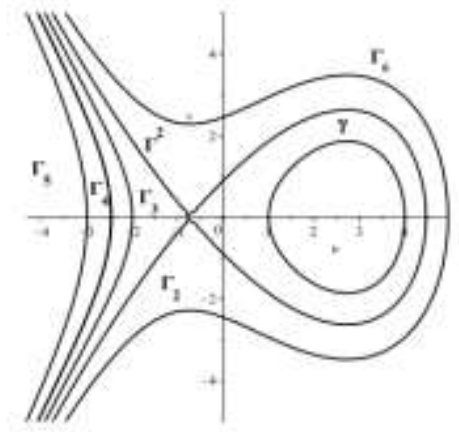

(a) $a=1, c=1, e=1$

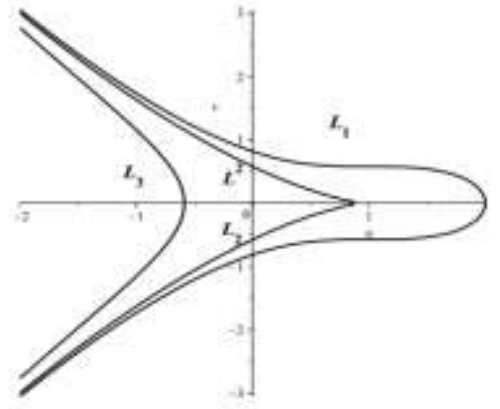

(b) $a=1, c=1, e=-\frac{1}{2}$

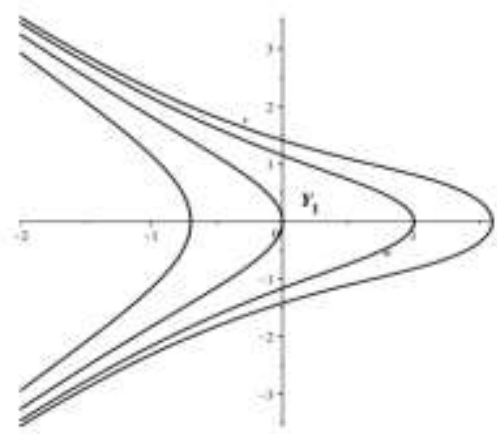

(c) $a=1, c=1, e=-1$

Fig-1: The phase portraits of $(2.5)$

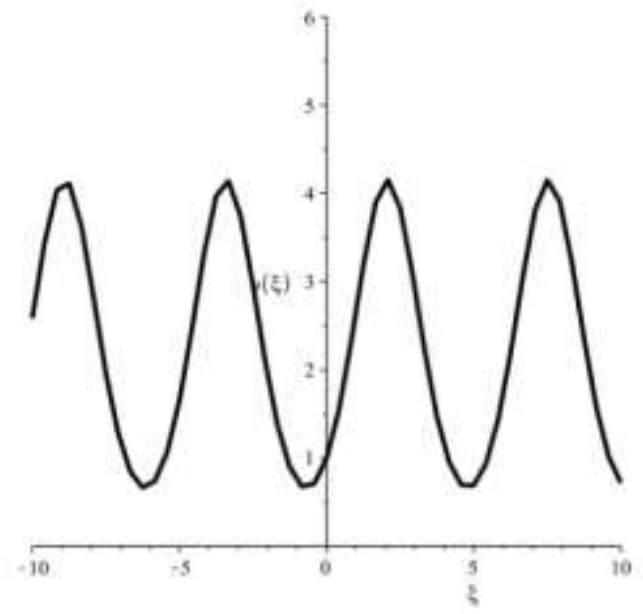

(a) $a=1, b=\frac{1}{3}, c=1, e=1, u(0)=1, u^{\prime}(0)=$ 1.

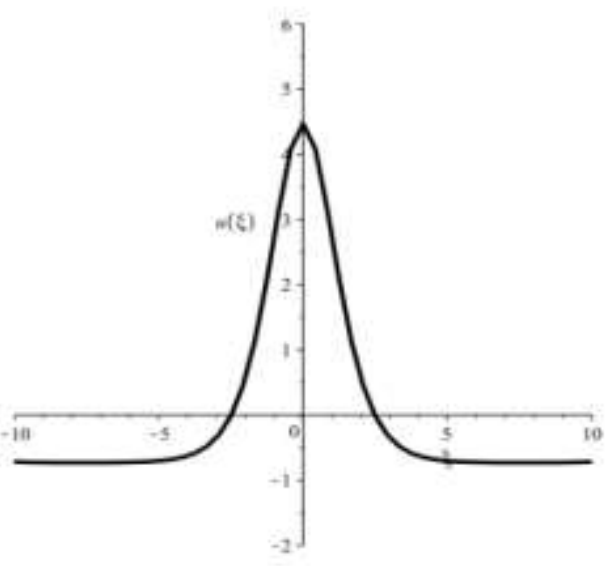

(b) $a=1, b=\frac{1}{3}, c=1, e=1, u(0)=$ $4.464100806, u^{\prime}(0)=0$.

Fig-2: Simulations of periodic wave and solitary wave

Finally, we apply numerical simulation method to verify the correction of the bifurcation results and to demonstrate wave shapes of various bounded and unbounded traveling waves of system (2.5). By fixing parameters in different parameter bifurcation sets and taking proper initial values, we simulate two types of bounded traveling waves Figure2.

\section{Explicit traveling wave solutions of Eq. (1.1)}

In this section, we will give the explicit expression of all bounded traveling wave solutions for Eq. (1.1), 
Consider the periodic orbits, from the energy function (2.4), any one of the periodic orbits $\Gamma(h)$ can be expressed by

$$
v= \pm \sqrt{\frac{a}{9 b}} \sqrt{\left(u-u_{1}\right)\left(u-u_{2}\right)\left(u_{3}-u\right)}
$$

Where $u_{1}, u_{2}$ and $u_{3}$ are reals and $u_{1}<u_{2}<u<u_{3}$. Assume that the period of these closed orbits is 2T, and choose $u(0)=u_{2}$, we have

$$
\begin{gathered}
\int_{u_{2}}^{u} \frac{d u}{\sqrt{\frac{a}{9 b}} \sqrt{\left(u-u_{1}\right)\left(u-u_{2}\right)\left(u_{3}-u\right)}}=\int_{0}^{\xi} d \xi, 0<\xi<T . \\
-\int_{u}^{u_{2}} \frac{d u}{\sqrt{\frac{a}{9 b}} \sqrt{\left(u-u_{1}\right)\left(u-u_{2}\right)\left(u_{3}-u\right)}}=\int_{\xi}^{0} d \xi,-T<\xi<0 .
\end{gathered}
$$

which can be rewritten as

$$
\int_{u_{2}}^{u} \frac{d u}{\sqrt{\frac{a}{9 b}} \sqrt{\left(u-u_{1}\right)\left(u-u_{2}\right)\left(u_{3}-u\right)}}=|\xi|,-T<\xi<T .
$$

By calculating the elliptic integral

$$
\int_{u_{2}}^{u} \frac{d p}{\sqrt{\left(u-u_{1}\right)\left(u-u_{2}\right)\left(u_{3}-u\right)}}=g \cdot s n^{-1}\left(\sqrt{\frac{\left(u_{3}-u_{1}\right)\left(u-u_{2}\right)}{\left(u_{3}-u_{2}\right)\left(u-u_{1}\right)}}, k\right),
$$

Where $g=\frac{2}{\sqrt{u_{3}-u_{1}}}$ and $k^{2}=\frac{u_{3}-u_{2}}{u_{3}-u_{1}}$, we get the expression of periodic wave solution of the system (2.4)

$$
u_{1}(\xi)=u_{1}+\frac{\left(u_{2}-u_{1}\right)\left(u_{3}-u_{1}\right)}{\left(u_{3}-u_{1}\right)-\left(u_{3}-u_{2}\right) \cdot \operatorname{sn}^{2}\left(\sqrt{\frac{a\left(u_{3}-u_{1}\right)}{36 b}} \xi\right)},-T<\xi<T \text {. (3.1) }
$$

Consider the homologous orbit, by (2.5), the homologous orbit $\gamma$ can be expressed by

$$
v= \pm \sqrt{\frac{a}{9 b}} \sqrt{\left(u-u_{4}\right)^{2}\left(u_{5}-u\right)}= \pm \sqrt{\frac{a}{9 b}}\left(u-u_{4}\right) \sqrt{u_{5}-u}
$$

Where $u_{4}=\frac{c-\sqrt{c^{2}+2 a e}}{a}$ and $u_{5}=\frac{c+2 \sqrt{c^{2}+2 a e}}{a}$ satisfy the condition that $u_{4}<u<u_{5}$. Choosing initial value $u(0)=u_{5}$, we have

$$
\begin{gathered}
\int_{u}^{u_{5}} \frac{d u}{\sqrt{\frac{a}{9 b}}\left(u-u_{4}\right) \sqrt{u_{5}-u},}=\int_{\xi}^{0} d \xi, \xi<0, \\
-\int_{u_{5}}^{u} \frac{d u}{\sqrt{\frac{a}{9 b}}\left(u-u_{4}\right) \sqrt{u_{5}-u}}=\int_{0}^{\xi} d \xi, \xi>0,
\end{gathered}
$$

Which can be rewritten as

$$
\int_{u_{5}}^{u} \frac{d u}{\sqrt{\frac{a}{9 b}}\left(u-u_{4}\right) \sqrt{u_{5}-u}}=-|\xi|,-\infty<\xi<+\infty .
$$

Noting that

$$
\int_{u_{5}}^{u} \frac{d u}{\left(u-u_{4}\right) \sqrt{u_{5}-u}}=\frac{1}{\sqrt{u_{5}-u_{4}}} \ln \frac{\sqrt{u_{5}-u_{4}}-\sqrt{u_{5}-u}}{\sqrt{u_{5}-u_{4}}+\sqrt{u_{5}-u}}
$$


We get the expression of solitary wave solution of the system (2.4)

$$
u_{2}(\xi)=u_{5}-\frac{\left(u_{5}-u_{4}\right)\left(1-\exp \left(\sqrt{\frac{a}{9 b}\left(u_{5}-u_{4}\right)}|\xi|\right)\right)^{2}}{\left(1+\exp \left(\sqrt{\frac{a}{9 b}\left(u_{5}-u_{4}\right)|\xi|}\right)\right)^{2}},-\infty<\xi<+\infty \text {. }
$$

It's easy to check that $u_{2}(\xi)=u_{2}(-\xi)$, It means that $u_{2}(\xi)$ can be simplified to the following form

$$
u_{2}(\xi)=u_{5}-\frac{\left(u_{5}-u_{4}\right)\left(1-\exp \left(\sqrt{\frac{a}{9 b}\left(u_{5}-u_{4}\right) \xi}\right)\right)^{2}}{\left(1+\exp \left(\sqrt{\frac{a}{9 b}\left(u_{5}-u_{4}\right) \xi}\right)\right)^{2}},-\infty<\xi<+\infty \text {. }
$$

\section{CONCLUSIONS}

In this paper, we apply the bifurcation method of dynamical system to study all types of traveling waves of the $(3+1)$-dimensional Zakharov-Kuznetsov equation thoroughly. Our results show that bounded traveling wave solutions of the $(3+1)$-dimensional Zakharov-Kuznetsov equation $(\mathrm{n}=1)$ can be divided into two types, including the periodic wave solution and the solitary wave solution. Moreover, when $\mathrm{n}$ is odd, bounded traveling wave solutions of the $(3+1)$ dimensional Zakharov-Kuznetsov equation is similar to the expression of $(n=1)$.

\section{REFERENCES}

1. Dong, Z. Z., Chen, Y., \& Lang, Y. H. (2010). Symmetry reduction and exact solutions of the $(3+$ 1)-dimensional ZakharovCKuznetsov equation. Chinese Physics B. 19:090205(1-7).

2. Zhang, W. B., \& Zhou, J. B. (2012). Traveling Wave Solutions of a Generalized ZakharovKuznetsov Equation. ISRN Mathematical Analysis. 2012:1-10.

3. Cui, C. L., \& Huang, S. Y. (2013). New interaction solutions of $(3+1)$-dimensional ZakharovCKuznetsov equation. Indian Journal of Physics. 87:897-901.

4. Sahoo, S., \& Saha Ray, S. (2015). Improved fractional sub-equation method for $(3+1)$ dimensional generalized fractional KdVCZakharovCKuznetsov equations. Computers Mathematics with Applications. 70:158-166.

5. Moleleki, L. D., Muatjetjeja, B., \& Adem, A. R. (2017). Solutions and conservation laws of a (3+ 1)-dimensional ZakharovCKuznetsov equation. Nonlinear Dynamics. 87:2187-2192.

6. Lu, D. C., Seadawy, A. R., Arshad, M., \& Wang, J. (2017). New solitary wave solutions of $(3+1)$ dimensional nonlinear extended Zakharov-
Kuznetsov and modified KdV-ZakharovKuznetsov equations and their applications. Results in physics. 7:899-909.

7. Korkmaz, A. (2017). Exact solutions to $(3+1)$ conformable time fractional JimboCMiwa, ZakharovCKuznetsov and modified ZakharovCKuznetsov equations. Communications in Theoretical Physics. 67:479-482.

8. Seadawy, A. R., \& Lu, D. (2016). Ion acoustic solitary wave solutions of three-dimensional nonlinear extended Zakharov-Kuznetsov dynamical equation in a magnetized two-iontemperature dusty plasma. Results in physics, 6 , 590-593.

9. Senyue, L. (1997). Higher-dimensional integrable models with a common recursion operator. Commun Theor Phys. 28(1):41-50.

10. Xu, G. Q., Zhang, S. Q., \& Li., Z. B. (2003). A simplified hirota method and its application. Journal Shanghai Univ. 7(2):143-147.

11. Wazwaz, A. M. (2010). Integrable (2+1)dimensional and (3+1)-dimensional breaking soliton equations. Phys Scr. 81(3): 035005.

12. Wazwaz, A. M. (2010). Multiple soliton solutions for the Bogoyavlenskii's generalized breaking soliton equations and its extension form. Appl Math Comput. 217(8):4282-4288.

13. Guckenheimer, J., \& Holmes, P. (1983). Nonlinear Oscillations, Dynamical systems and bifurcation of vector fields, Springer, New York, NY.

14. Chow, S. N., Hale, J. K. (2012). Methods of bifurcation theory, Springer Science \& Business Media.

15. Zhang, Z. F., Ding, T. R., Huang, W. Z., \& Dong, Z. X. (1992). Qualitative theory of differential equations, American Mathematical Society, Providence, RI, USA. 